\title{
Superfluidity in Dilute Trapped Bose Gases
}

\author{
Elliott H. Lieb ${ }^{1, *}$, Robert Seiringer ${ }^{1,2, \dagger}$, and Jakob Yngvason ${ }^{2}$ \\ 1. Department of Physics, Jadwin Hall, Princeton University, \\ P. O. Box 708, Princeton, New Jersey 08544 \\ 2. Institut für Theoretische Physik, Universität Wien, \\ Boltzmanngasse 5, A-1090 Vienna, Austria
}

May 27, 2002

\begin{abstract}
A commonly used theoretical definition of superfluidity in the ground state of a Bose gas is based on the response of the system to an imposed velocity field or, equivalently, to twisted boundary conditions in a box. We are able to carry out this program in the case of a dilute interacting Bose gas in a trap. We prove that a gas with repulsive interactions is $100 \%$ superfluid in the dilute limit in which the Gross-Pitaevskii equation is exact. This is the first example in an experimentally realistic continuum model in which superfluidity is rigorously verified.
\end{abstract}

\section{Introduction}

The phenomenological two-fluid model of superfluidity (see, e.g., [1]) is based on the idea that the particle density $\rho$ is composed of two parts, the density $\rho_{\mathrm{s}}$ of the inviscid superfluid and the normal fluid density $\rho_{\mathrm{n}}$. If an external velocity field is imposed on the fluid (for instance by moving the walls of the container) only the viscous normal component responds to the velocity field, while the superfluid component stays at rest. In accord with these ideas the superfluid density in the ground state is commonly defined as follows [2]: Let $E_{0}$ denote the ground state energy of the system in the rest frame and $E_{0}^{\prime}$ the ground state energy, measured in the moving frame, when a velocity field $\mathbf{v}$ is imposed. Then for small $\mathbf{v}$

$$
\frac{E_{0}^{\prime}}{N}=\frac{E_{0}}{N}+\left(\rho_{\mathrm{s}} / \rho\right) \frac{1}{2} m \mathbf{v}^{2}+O\left(|\mathbf{v}|^{4}\right)
$$

where $N$ is the particle number and $m$ the particle mass. At positive temperatures the ground state energy should be replaced by the free energy. (Remark: It is important here that (1) holds uniformly for all large $N$. For fixed $N$ and a finite

\footnotetext{
${ }^{*}$ Work partially supported by U.S. National Science Foundation grant PHY 98-20650.

${ }^{\dagger}$ Erwin Schrödinger Fellow, supported by the Austrian Science Fund.

(c) 2002 by the authors. This paper may be reproduced, in its entirety, for non-commercial purposes.
} 
box, Eq. (四) with $\rho_{\mathrm{s}} / \rho=1$ always holds for a Bose gas with an arbitrary interaction if $\mathbf{v}$ is small enough, owing to the discreteness of the energy spectrum [3].) There are other definitions of the superfluid density that may lead to slightly different results [4], but this is the one we shall use. By a unitary gauge transformation the passage from $E_{0}$ to $E_{0}^{\prime}$ is equivalent to replacing periodic boundary conditions in a box by twisted boundary conditions in the direction of the velocity field (see below).

One of the unresolved issues in the theory of superfluidity is its relation to BoseEinstein condensation (BEC). It has been argued that in general neither condition is necessary for the other (c.f., e.g., [5]). A simple example illustrating the fact that BEC is not necessary for superfluidity is the 1D hard-core Bose gas. This system is well known to have a spectrum like that of an ideal Fermi gas, and it is easy to see that it is superfluid in its ground state in the sense of (11). On the other hand, it has no BEC [6].

Recently, BEC has been rigorously established for the first time for a physically realistic many-body Hamiltonian [7] and it is clearly of interest to know whether superfluidity is also exhibited in this model. The purpose of this letter is to show that this is indeed the case: We prove that the ground state of a Bose gas with short range, repulsive interaction is $100 \%$ superfluid in the dilute limit in which the Gross-Pitaevskii (GP) description of the gas is exact. This is the limit in which the particle number tends to infinity, but the ratio $N a / L$, where $a$ is the scattering length of the interaction potential and $L$ the box size, is kept fixed. (The significance of the parameter $N a / L$ is that it is the ratio of the ground state energy per particle, $\sim N a / L^{3}$, to the lowest excitation energy in the box, $\sim 1 / L^{2}$.) In addition we show that the gas remains $100 \%$ Bose-Einstein condensed in this limit, also for a finite velocity v. Both results can be generalized from periodic boxes to (non-constant) velocity fields in a cylindrical geometry.

We wish to emphasize that in this GP limit the fact that there is $100 \%$ condensation does not mean that no significant interactions occur. The kinetic and potential energies can differ markedly from that obtained with a simple variational function that is an $N$-fold product of one-body condensate wave functions. This assertion might seem paradoxical, and the explanation is that near the GP limit the region in which the wave function differs from the condensate function has a tiny volume that goes to zero as $N \rightarrow \infty$. Nevertheless, the interaction energy, which is proportional to $N$, resides in this tiny volume.

\section{$2 \quad$ Setting and Main Results}

We consider a Bose gas with the Hamiltonian

$$
H_{N}=-\mu \sum_{j=1}^{N} \nabla_{j}^{2}+\sum_{1 \leq i<j \leq N} v\left(\left|\mathbf{r}_{i}-\mathbf{r}_{j}\right|\right),
$$

where $\mu=\hbar^{2} /(2 m)$ and the interaction potential $v$ is nonnegative and of finite range. The two-body scattering length of $v$ is denoted by $a$. The Hamiltonian acts on totally 
symmetric functions $\Psi$ of $N$ variables $\mathbf{r}_{i}=\left(x_{i}, y_{i}, z_{i}\right) \in \mathcal{K} \subset \mathbb{R}^{3}$, where $\mathcal{K}$ denotes the cube $[0, L]^{3}$ of side length $L$. (We could easily use a cuboid of sides $L_{1}, L_{2}, L_{3}$ instead.) Initially we assume periodic boundary conditions in all three coordinate directions.

Imposing an external velocity field $\mathbf{v}=(0,0, \pm|\mathbf{v}|)$ means that the momentum operator $\mathbf{p}=-\mathrm{i} \hbar \nabla$ is replaced by by $\mathbf{p}-m \mathbf{v}$, but retaining the periodic boundary conditions. By a unitary gauge transformation,

$$
(U \Psi)\left(\mathbf{r}_{1}, \ldots, \mathbf{r}_{N}\right)=e^{\mathrm{i} \varphi\left(\sum_{i} z_{i}\right) / L} \Psi\left(\mathbf{r}_{1}, \ldots, \mathbf{r}_{N}\right),
$$

this is equivalent to leaving the Hamiltonian intact, but changing the periodic boundary condition in the $z$-direction to the twisted boundary condition

$$
\Psi\left(\mathbf{r}_{1}+(0,0, L), \mathbf{r}_{2}, \ldots, \mathbf{r}_{N}\right)=e^{\mathrm{i} \varphi} \Psi\left(\mathbf{r}_{1}, \mathbf{r}_{2}, \ldots, \mathbf{r}_{N}\right)
$$

where the dimensionless phase $\varphi$ is connected to the velocity $\mathbf{v}$ by

$$
\varphi=\frac{ \pm|\mathbf{v}| L m}{\hbar} .
$$

Let $E_{0}(N, a, \varphi)$ denote the ground state energy of (2) with the boundary condition (4). Obviously it is no restriction to consider only the case $-\pi \leq \varphi \leq \pi$, since $E_{0}$ is periodic in $\varphi$ with period $2 \pi$. For $\Psi_{0}$ the ground state of $H_{N}$, let $\gamma_{N}$ be its one-particle reduced density matrix

$$
\gamma_{N}\left(\mathbf{r}, \mathbf{r}^{\prime}\right)=N \int_{\mathcal{K}^{N-1}} \Psi_{0}\left(\mathbf{r}, \mathbf{r}_{2}, \ldots, \mathbf{r}_{N}\right) \Psi_{0}^{*}\left(\mathbf{r}^{\prime}, \mathbf{r}_{2}, \ldots, \mathbf{r}_{N}\right) d \mathbf{r}_{2} \cdots d \mathbf{r}_{N}
$$

We are interested in the Gross-Pitaevskii (GP) limit $N \rightarrow \infty$ with $N a / L$ fixed. We also fix the box size $L$. This means that $a$ should vary like $1 / N$ which can be achieved by writing $v(\mathbf{r})=a^{-2} v_{1}(\mathbf{r} / a)$, where $v_{1}$ is a fixed potential with scattering length 1 , while $a$ changes with $N$.

Theorem 1 (Superfluidity of the homogeneous Bose gas). For $|\varphi| \leq \pi$

$$
\lim _{N \rightarrow \infty} \frac{E_{0}(N, a, \varphi)}{N}=4 \pi \mu a \rho+\mu \frac{\varphi^{2}}{L^{2}}
$$

in the limit $N \rightarrow \infty$ with $N a / L$ and $L$ fixed. Here $\rho=N / L^{3}$, so a $\rho$ is fixed too. In the same limit, for $|\varphi|<\pi$,

$$
\lim _{N \rightarrow \infty} \frac{1}{N} \gamma_{N}\left(\mathbf{r}, \mathbf{r}^{\prime}\right)=\frac{1}{L^{3}} e^{\mathrm{i} \varphi\left(z-z^{\prime}\right) / L}
$$

in trace class norm, i.e., $\lim _{N \rightarrow \infty}$ Trace $\left[\left|\gamma_{N} / N-\right| L^{-3 / 2} e^{i \varphi z}\right\rangle\left\langle L^{-3 / 2} e^{i \varphi z}||\right]=0$.

Note that, by the definition (可) of $\rho_{s}$ and Eq. (5), Eq. (7) means that $\rho_{s}=\rho$, i.e., there is $100 \%$ superfluidity. For $\varphi=0$, Eq. (7) was first proved in [8]. Eq. (8) for $\varphi=0$ is the BEC proved in [7. 
The reason that we have to restrict ourselves to $|\varphi|<\pi$ in the second part of Theorem 1 is that for $|\varphi|=\pi$ there are two ground states of the operator $-\nabla^{2}$ with the twisted boundary conditions (田). All we can say in this case is that there is a subsequence of $\gamma_{N}$ that converges to a density matrix of rank $\leq 2$, whose range is spanned by these two functions.

Theorem 1 can be generalized in various ways to a physically more realistic setting. As an example, let $\mathcal{K}$ be a finite cylinder based on an annulus centered at the origin. Given a bounded, real function $a(r, z)$ let $A$ be the vector field (in polar coordinates) $A(r, \theta, z)=\varphi a(r, z) \widehat{e}_{\theta}$, where $\widehat{e}_{\theta}$ is the unit vector in the $\theta$ direction. We also allow for a bounded external potential $V(r, z)$ that does not depend on $\theta$.

Using the methods of Appendix A in [9], it is not difficult to see that there exists a $\varphi_{0}>0$, depending only on $\mathcal{K}$ and $a(r, z)$, such that for all $|\varphi|<\varphi_{0}$ there is a unique minimizer $\phi^{\mathrm{GP}}$ of the Gross-Pitaevskii functional

$$
\mathcal{E}^{\mathrm{GP}}[\phi]=\int_{\mathcal{K}}\left(\mu|(\nabla+\mathrm{i} A(\mathbf{r})) \phi(\mathbf{r})|^{2}+V(\mathbf{r})|\phi(\mathbf{r})|^{2}+4 \pi \mu N a|\phi(\mathbf{r})|^{4}\right) d^{3} \mathbf{r}
$$

under the normalization condition $\int|\phi|^{2}=1$. This minimizer does not depend on $\theta$, and can be chosen to be positive, for the following reason: The relevant term in the kinetic energy is $T=-r^{-2}[\partial / \partial \theta+\mathrm{i} \varphi r a(r, z)]^{2}$. If $|\varphi r a(r, z)|<1 / 2$, it is easy to see that $T \geq \varphi^{2} a(r, z)^{2}$, in which case, without raising the energy, we can replace $\phi$ by the square root of the $\theta$-average of $|\phi|^{2}$. This can only lower the kinetic energy 110] and, by convexity of $x \rightarrow x^{2}$, this also lowers the $\phi^{4}$ term.

We denote the ground state energy of $\mathcal{E}^{\mathrm{GP}}$ by $E^{\mathrm{GP}}$, depending on $N a$ and $\varphi$. The following Theorem 2 concerns the ground state energy $E_{0}$ of

$$
H_{N}=\sum_{j=1}^{N}\left[-\mu\left(\nabla_{j}+\mathrm{i} A\left(\mathbf{r}_{j}\right)\right)^{2}+V\left(\mathbf{r}_{j}\right)\right]+\sum_{1 \leq i<j \leq N} v\left(\left|\mathbf{r}_{i}-\mathbf{r}_{j}\right|\right),
$$

with Neumann boundary conditions on $\mathcal{K}$, and the one-particle reduced density matrix $\gamma_{N}$ of the ground state, respectively. Different boundary conditions can be treated in the same manner, if they are also used in (9).

Theorem 2 (Superfluidity in a cylinder). For $|\varphi|<\varphi_{0}$

$$
\lim _{N \rightarrow \infty} \frac{E_{0}(N, a, \varphi)}{N}=E^{\mathrm{GP}}(N a, \varphi)
$$

in the limit $N \rightarrow \infty$ with $N$ a fixed. In the same limit,

$$
\lim _{N \rightarrow \infty} \frac{1}{N} \gamma_{N}\left(\mathbf{r}, \mathbf{r}^{\prime}\right)=\phi^{\mathrm{GP}}(\mathbf{r}) \phi^{\mathrm{GP}}\left(\mathbf{r}^{\prime}\right)
$$

in trace class norm, i.e., $\lim _{N \rightarrow \infty}$ Trace $\left[\left|\gamma_{N} / N-\right| \phi^{\mathrm{GP}}\right\rangle\left\langle\phi^{\mathrm{GP}}||\right]=0$.

To illustrate the nature of $\varphi_{0}$, consider the special case of the curl-free vector potential $A(r, \theta)=\varphi r^{-1} \widehat{e}_{\theta}$, i.e., $a(r, z)=r^{-1}$. In this case, there is a unique GP minimizer for all $\varphi \notin \mathbb{Z}+\frac{1}{2}$, whereas there are two minimizers for $\varphi \in \mathbb{Z}+\frac{1}{2}$. Part two of Theorem 2 holds in this special case for all $\varphi \notin \mathbb{Z}+\frac{1}{2}$, and (11) is true even for all $\varphi$. 


\section{Proofs}

In the following, we will present only a proof of Theorem 1 for simplicity. Theorem 2 can be proved using the same methods, and additionally the methods of [7] to deal with the inhomogeneity of the system.

Proof of Theorem 11. We start by making the inverse unitary transformation $U^{*}$, with $U$ given in (3). The wave function $U^{*} \Psi$ obtained this way then satisfies periodic boundary conditions on $\mathcal{K}$ if $\Psi$ satisfies the twisted boundary condition (雨). Moreover, the Hamiltonian transforms as

$$
U^{*} H_{N} U=-\mu \sum_{j=1}^{N}\left(\nabla_{j}+\mathrm{i} \varphi / L\right)^{2}+\sum_{1 \leq i<j \leq N} v\left(\left|\mathbf{r}_{i}-\mathbf{r}_{j}\right|\right) \equiv \widetilde{H}_{N},
$$

where we denoted for short $\varphi=(0,0, \varphi)$.

As in [8] we define $Y=(4 \pi / 3) \rho a^{3}$. Note that in the limit considered, $Y \sim N^{-2}$. We first consider with the upper bound to $E_{0}$. Using the ground state $\Psi_{0}$ for $\varphi=0$ as a trial function, we immediately get

$$
E_{0}(N, a, \varphi) \leq\left\langle\Psi_{0}, \widetilde{H}_{N} \Psi_{0}\right\rangle=E_{0}(N, a, 0)+N \mu \frac{\varphi^{2}}{L^{2}},
$$

since $\left\langle\Psi_{0}, \nabla_{i} \Psi_{0}\right\rangle=0$. From [9] we know that $E_{0}(N, a, 0) \leq 4 \pi \mu N \rho a\left(1+\right.$ const. $\left.Y^{1 / 3}\right)$, which has the right form as $N \rightarrow \infty$.

For the lower bound to the ground state energy we need the following Lemma.

Lemma 1 (Localization of energy). For all symmetric, normalized wave functions $\Psi\left(\mathbf{r}_{1}, \ldots, \mathbf{r}_{N}\right)$ with periodic boundary conditions on $\mathcal{K}$, and for $N \geq Y^{-1 / 17}$,

$$
\begin{aligned}
\frac{1}{N}\left\langle\Psi, \widetilde{H}_{N} \Psi\right\rangle \geq & \left(1-\text { const. } Y^{1 / 17}\right) \\
& \times\left(4 \pi \mu \rho a+\mu \int_{\mathcal{K}^{N-1}} d \mathbf{X} \int_{\Omega_{\mathbf{X}}} d \mathbf{r}_{1}\left|\left(\nabla_{1}+\mathrm{i} \varphi / L\right) \Psi\left(\mathbf{r}_{1}, \mathbf{X}\right)\right|^{2}\right),
\end{aligned}
$$

where $\mathbf{X}=\left(\mathbf{r}_{2}, \ldots, \mathbf{r}_{N}\right), d \mathbf{X}=\prod_{j=2}^{N} d \mathbf{r}_{j}$, and

$$
\Omega_{\mathbf{X}}=\left\{\mathbf{r}_{1}: \min _{j \geq 2}\left|\mathbf{r}_{1}-\mathbf{r}_{j}\right| \geq R\right\}
$$

with $R=a Y^{-5 / 17}$.

Proof. Since $\Psi$ is symmetric, the left side of (15) can be written as

$$
\int_{\mathcal{K}^{N-1}} d \mathbf{X} \int_{\mathcal{K}} d \mathbf{r}_{1}\left[\mu\left|\left(\nabla_{1}+\mathrm{i} \boldsymbol{\varphi} / L\right) \Psi\left(\mathbf{r}_{1}, \mathbf{X}\right)\right|^{2}+\frac{1}{2} \sum_{j \geq 2} v\left(\left|\mathbf{r}_{1}-\mathbf{r}_{j}\right|\right)\left|\Psi\left(\mathbf{r}_{1}, \mathbf{X}\right)\right|^{2}\right] .
$$

For any $\varepsilon>0$ and $R>0$ this is

$$
\geq \varepsilon T+(1-\varepsilon)\left(T^{\mathrm{in}}+I\right)+(1-\varepsilon) T_{\varphi}^{\mathrm{out}},
$$


with

$$
\begin{gathered}
T=\mu \int_{\mathcal{K}^{N-1}} d \mathbf{X} \int_{\mathcal{K}} d \mathbf{r}_{1}\left|\nabla_{1}\right| \Psi\left(\mathbf{r}_{1}, \mathbf{X}\right)||^{2} \\
T^{\text {in }}=\left.\mu \int_{\mathcal{K}^{N-1}} d \mathbf{X} \int_{\Omega_{\mathbf{X}}^{c}} d \mathbf{r}_{1}\left|\nabla_{1}\right| \Psi\left(\mathbf{r}_{1}, \mathbf{X}\right)\right|^{2}, \\
T_{\varphi}^{\text {out }}=\mu \int_{\mathcal{K}^{N-1}} d \mathbf{X} \int_{\Omega_{\mathbf{X}}} d \mathbf{r}_{1}\left|\left(\nabla_{1}+\mathrm{i} \varphi / L\right) \Psi\left(\mathbf{r}_{1}, \mathbf{X}\right)\right|^{2},
\end{gathered}
$$

and

$$
I=\frac{1}{2} \int_{\mathcal{K}^{N-1}} d \mathbf{X} \int_{\mathcal{K}} d \mathbf{r}_{1} \sum_{j \geq 2} v\left(\left|\mathbf{r}_{1}-\mathbf{r}_{j}\right|\right)\left|\Psi\left(\mathbf{r}_{1}, \mathbf{X}\right)\right|^{2}
$$

Here

$$
\Omega_{\mathbf{X}}^{c}=\left\{\mathbf{r}_{1}:\left|\mathbf{r}_{1}-\mathbf{r}_{j}\right|<R \text { for some } j \geq 2\right\}
$$

is the complement of $\Omega_{\mathbf{X}}$, and the diamagnetic inequality $|(\nabla+i \varphi / L) f(\mathbf{r})|^{2} \geq$ $|\nabla| f(\mathbf{r}) \|^{2}$ has been used. The proof is completed by using the results of $[\mathbb{8}]$ and [7] (see also [11) which tell us that for $\varepsilon=Y^{1 / 17}$ and $R=a Y^{-5 / 17}$

$$
\varepsilon T+(1-\varepsilon)\left(T^{\text {in }}+I\right) \geq\left(1-\text { const. } Y^{1 / 17}\right) 4 \pi \mu \rho a
$$

as long as $N \geq Y^{-1 / 17}$.

The following Lemma 2 is needed for a lower bound on the second term in (15). It is stated for $\mathcal{K}$ the torus $[0, L]^{3}$ with opposite sides identified, but it can be generalized to arbitrary connected sets $\mathcal{K}$ that are sufficiently nice so that the Rellich-Kondrashov Theorem (see [10, Thm. 8.9]) holds on $\mathcal{K}$. In particular, this is the case if $\mathcal{K}$ has the 'cone property' 10]. Another possible generalization is to include general bounded vector fields replacing $\varphi$, see [12].

If $\Omega$ is any subset of $\mathcal{K}$ we shall denote $\int_{\Omega} f^{*}(\mathbf{r}) g(\mathbf{r}) d \mathbf{r}$ by $\langle f, g\rangle_{\Omega}$ and $\langle f, f\rangle_{\Omega}^{1 / 2}$ by $\|f\|_{L^{2}(\Omega)}$. We also denote $\nabla+\mathrm{i} \varphi$ by $\nabla_{\varphi}$ for short.

Lemma 2 (Generalized Poincaré inequality). For any $|\varphi|<\pi$ there are constants $c>0$ and $C<\infty$ such that for all subsets $\Omega \subset \mathcal{K}$ and all functions $f$ on the torus $\mathcal{K}$ the following estimate holds:

$$
\begin{aligned}
\left\|\nabla_{\varphi} f\right\|_{L^{2}(\Omega)}^{2} \geq \frac{\varphi^{2}}{L^{2}}\|f\|_{L^{2}(\mathcal{K})}^{2}+ & \frac{c}{L^{2}}\left\|f-L^{-3}\langle 1, f\rangle_{\mathcal{K}}\right\|_{L^{2}(\mathcal{K})}^{2} \\
& -C\left(\left\|\nabla_{\varphi} f\right\|_{L^{2}(\mathcal{K})}^{2}+\frac{1}{L^{2}}\|f\|_{L^{2}(\mathcal{K})}^{2}\right)\left(\frac{|\Omega|^{c}}{|\mathcal{K}|}\right)^{1 / 2} .
\end{aligned}
$$

Here $\left|\Omega^{c}\right|$ is the volume of $\Omega^{c}=\mathcal{K} \backslash \Omega$, the complement of $\Omega$ in $\mathcal{K}$. 
Proof. We shall derive (25) from a special form of this inequality that holds for all functions that are orthogonal to the constant function. Namely, for any positive $\alpha<2 / 3$ and some constants $c>0$ and $\widetilde{C}<\infty$ (depending only on $\alpha$ and $|\varphi|<\pi$ ) we claim that

$$
\left\|\nabla_{\varphi} h\right\|_{L^{2}(\Omega)}^{2} \geq \frac{\varphi^{2}+c}{L^{2}}\|h\|_{L^{2}(\mathcal{K})}^{2}-\widetilde{C}\left(\frac{\left|\Omega^{c}\right|}{|\mathcal{K}|}\right)^{\alpha}\left\|\nabla_{\varphi} h\right\|_{L^{2}(\mathcal{K})}^{2},
$$

provided $\langle 1, h\rangle_{\mathcal{K}}=0$. (Remark: Eq. (26) holds also for $\alpha=2 / 3$, but the proof is slightly more complicated in that case. See [12].) If (26) is known the derivation of (25) is easy: For any $f$, the function $h=f-L^{-3}\langle 1, f\rangle_{\mathcal{K}}$ is orthogonal to 1 . Moreover,

$$
\begin{aligned}
\left\|\nabla_{\varphi} h\right\|_{L^{2}(\Omega)}^{2}= & \left\|\nabla_{\varphi} h\right\|_{L^{2}(\mathcal{K})}^{2}-\left\|\nabla_{\varphi} h\right\|_{L^{2}\left(\Omega^{c}\right)}^{2} \\
= & \left\|\nabla_{\varphi} f\right\|_{L^{2}(\Omega)}^{2}-\frac{\varphi^{2}}{L^{2}}\left|\left\langle L^{-3 / 2}, f\right\rangle_{\mathcal{K}}\right|^{2}\left(1+\frac{\left|\Omega^{c}\right|}{|\mathcal{K}|}\right) \\
& +2 \frac{\varphi}{L} \operatorname{Re}\left\langle L^{-3 / 2}, f\right\rangle_{\mathcal{K}}\left\langle\nabla_{\varphi} f, L^{-3 / 2}\right\rangle_{\Omega^{c}} \\
\leq & \left\|\nabla_{\varphi} f\right\|_{L^{2}(\Omega)}^{2}-\frac{\varphi^{2}}{L^{2}}\left|\left\langle L^{-3 / 2}, f\right\rangle_{\mathcal{K}}\right|^{2} \\
& +\frac{|\varphi|}{L}\left(L\left\|\nabla_{\varphi} f\right\|_{L^{2}(\mathcal{K})}^{2}+\frac{1}{L}\|f\|_{L^{2}(\mathcal{K})}^{2}\right)\left(\frac{\left|\Omega^{c}\right|}{|\mathcal{K}|}\right)^{1 / 2}
\end{aligned}
$$

and

$$
\frac{\varphi^{2}+c}{L^{2}}\|h\|_{L^{2}(\mathcal{K})}^{2}=\frac{\varphi^{2}}{L^{2}}\left(\|f\|_{L^{2}(\mathcal{K})}^{2}-\left|\left\langle L^{-3 / 2}, f\right\rangle_{\mathcal{K}}\right|^{2}\right)+\frac{c}{L^{2}}\left\|f-L^{-3}\langle 1, f\rangle_{\mathcal{K}}\right\|_{L^{2}(\mathcal{K})}^{2} .
$$

Setting $\alpha=\frac{1}{2}$, using $\left\|\nabla_{\varphi} h\right\|_{L^{2}(\mathcal{K})} \leq\left\|\nabla_{\varphi} f\right\|_{L^{2}(\mathcal{K})}$ in the last term in (26) and combining (26), (27) and (28) gives (25) with $C=|\varphi|+\widetilde{C}$.

We now turn to the proof of (26). For simplicity we set $L=1$. The general case follows by scaling. Assume that (26) is false. Then there exist sequences of constants $C_{n} \rightarrow \infty$, functions $h_{n}$ with $\left\|h_{n}\right\|_{L^{2}(\mathcal{K})}=1$ and $\left\langle 1, h_{n}\right\rangle_{\mathcal{K}}=0$, and domains $\Omega_{n} \subset \mathcal{K}$ such that

$$
\lim _{n \rightarrow \infty}\left\{\left\|\nabla_{\varphi} h_{n}\right\|_{L^{2}\left(\Omega_{n}\right)}^{2}+C_{n}\left|\Omega_{n}^{c}\right|^{\alpha}\left\|\nabla_{\varphi} h_{n}\right\|_{L^{2}(\mathcal{K})}^{2}\right\} \leq \varphi^{2}
$$

We shall show that this leads to a contradiction.

Since the sequence $h_{n}$ is bounded in $L^{2}(\mathcal{K})$ it has a subsequence, denoted again by $h_{n}$, that converges weakly to some $h \in L^{2}(\mathcal{K})$ (i.e., $\left\langle g, h_{n}\right\rangle_{\mathcal{K}} \rightarrow\langle g, h\rangle_{\mathcal{K}}$ for all $\left.g \in L^{2}(\mathcal{K})\right)$. Moreover, by Hölder's inequality the $L^{p}\left(\Omega_{n}^{c}\right)$ norm $\left\|\nabla_{\varphi} h_{n}\right\|_{L^{p}\left(\Omega_{n}^{c}\right)}=$ $\left(\int_{\Omega_{n}^{c}}|h(\mathbf{r})|^{p} d \mathbf{r}\right)^{1 / p}$ is bounded by $\left|\Omega_{n}^{c}\right|^{\alpha / 2}\left\|\nabla_{\varphi} h_{n}\right\|_{L^{2}(\mathcal{K})}$ for $p=2 /(\alpha+1)$. From (29) we conclude that $\left\|\nabla_{\varphi} h_{n}\right\|_{L^{p}\left(\Omega_{n}^{c}\right)}$ is bounded and also that $\left\|\nabla_{\varphi} h_{n}\right\|_{L^{p}\left(\Omega_{n}\right)} \leq\left\|\nabla_{\varphi} h_{n}\right\|_{L^{2}\left(\Omega_{n}\right)}$ is bounded. Altogether, $\nabla_{\varphi} h_{n}$ is bounded in $L^{p}(\mathcal{K})$, and by passing to a further subsequence if necessary, we can therefore assume that $\nabla_{\varphi} h_{n}$ converges weakly in $L^{p}(\mathcal{K})$. The same applies to $\nabla h_{n}$. Since $p=2 /(\alpha+1)$ with $\alpha<2 / 3$ the hypotheses 
of the Rellich-Kondrashov Theorem [10, Thm 8.9] are fulfilled and consequently $h_{n}$ converges strongly in $L^{2}(\mathcal{K})$ to $h$ (i.e., $\left\|h-h_{n}\right\|_{L^{2}(\mathcal{K})} \rightarrow 0$ ). We shall now show that

$$
\liminf _{n \rightarrow \infty}\left\|\nabla_{\varphi} h_{n}\right\|_{L^{2}\left(\Omega_{n}\right)}^{2} \geq\left\|\nabla_{\varphi} h\right\|_{L^{2}(\mathcal{K})}^{2} .
$$

This will complete the proof because the $h_{n}$ are normalized and orthogonal to 1 and the same holds for $h$ by strong convergence. Hence the right side of (30) is necessarily $>\varphi^{2}$, since for $|\varphi|<\pi$ the lowest eigenvalue of $-\nabla_{\varphi}^{2}$, with constant eigenfunction, is non-degenerate. This contradicts (29).

Eq. (30) is essentially a consequence of the weak lower semicontinuity of the $L^{2}$ norm, but the dependence on $\Omega_{n}$ leads to a slight complication. First, Eq. (29) and $C_{n} \rightarrow \infty$ clearly imply that $\left|\Omega_{n}^{c}\right| \rightarrow 0$, because $\left\|\nabla_{\varphi} h_{n}\right\|_{L^{2}(\mathcal{K})}^{2}>\varphi^{2}$. By choosing a subsequence we may assume that $\sum_{n}\left|\Omega_{n}^{c}\right|<\infty$. For some fixed $N$ let $\widetilde{\Omega}_{N}=$ $\mathcal{K} \backslash \cup_{n \geq N} \Omega_{n}^{c}$. Then $\tilde{\Omega}_{N} \subset \Omega_{n}$ for $n \geq N$. Since $\left\|\nabla_{\varphi} h_{n}\right\|_{L^{2}\left(\Omega_{n}\right)}^{2}$ is bounded, $\nabla_{\varphi} h_{n}$ is also bounded in $L^{2}\left(\widetilde{\Omega}_{N}\right)$ and a subsequence of it converges weakly in $L^{2}\left(\widetilde{\Omega}_{N}\right)$ to $\nabla_{\varphi} h$. Hence

$$
\liminf _{n \rightarrow \infty}\left\|\nabla_{\varphi} h_{n}\right\|_{L^{2}\left(\Omega_{n}\right)}^{2} \geq \liminf _{n \rightarrow \infty}\left\|\nabla_{\varphi} h_{n}\right\|_{L^{2}\left(\widetilde{\Omega}_{N}\right)}^{2} \geq\left\|\nabla_{\varphi} h\right\|_{L^{2}\left(\widetilde{\Omega}_{N}\right)}^{2} .
$$

Since $\widetilde{\Omega}_{N} \subset \widetilde{\Omega}_{N+1}$ and $\cup_{N} \widetilde{\Omega}_{N}=\mathcal{K}$ (up to a set of measure zero), we can now let $N \rightarrow \infty$ on the right side of (31). By monotone convergence this converges to $\left\|\nabla_{\varphi} h\right\|_{L^{2}(\mathcal{K})}^{2}$. This proves (30) which, as remarked above, contradicts (29).

We now are able to finish the proof of Theorem 1. From Lemmas 1 and 2 we infer that, for any symmetric $\Psi$ with $\langle\Psi, \Psi\rangle=1$ and for $N$ large enough,

$$
\begin{aligned}
\frac{1}{N}\left\langle\Psi, \widetilde{H}_{N} \Psi\right\rangle\left(1-\text { const. } Y^{1 / 17}\right)^{-1} \geq & \\
4 \pi \mu \rho a & +\mu \frac{\varphi^{2}}{L^{2}}-C Y^{1 / 17}\left(\frac{1}{L^{2}}+\frac{1}{N}\left\langle\Psi, \sum_{j}\left(\nabla_{j}+\mathrm{i} \varphi\right) \Psi\right\rangle\right) \\
& +\frac{c}{L^{2}} \int_{\mathcal{K}^{N-1}} d \mathbf{X} \int_{\mathcal{K}} d \mathbf{r}_{1}\left|\Psi\left(\mathbf{r}_{1}, \mathbf{X}\right)-L^{-3}\left[\int_{\mathcal{K}} d \mathbf{r} \Psi(\mathbf{r}, \mathbf{X})\right]\right|^{2}
\end{aligned}
$$

where we used that $\left|\Omega^{c}\right| \leq \frac{4 \pi}{3} N R^{3}=$ const. $L^{3} Y^{2 / 17}$. From this we can infer two things. First, since the kinetic energy, divided by $N$, is certainly bounded independent of $N$, as the upper bound shows, we get that

$$
\liminf _{N \rightarrow \infty} \frac{E_{0}(N, a, \varphi)}{N} \geq 4 \pi \mu \rho a+\mu \frac{\varphi^{2}}{L^{2}}
$$

for any $|\varphi|<\pi$. By continuity this holds also for $|\varphi|=\pi$, proving (7). (To be precise, $E_{0} / N-\mu \varphi^{2} L^{-2}$ is concave in $\varphi$, and therefore stays concave, and in particular continuous, in the limit $N \rightarrow \infty$.) Secondly, since the upper and the lower bound to $E_{0}$ agree in the limit considered, the positive last term in (32) has to vanish in the limit. I.e., we get that for the ground state wave function $\Psi_{0}$ of $\widetilde{H}_{N}$

$$
\lim _{N \rightarrow \infty} \int_{\mathcal{K}^{N-1}} d \mathbf{X} \int_{\mathcal{K}} d \mathbf{r}_{1}\left|\Psi_{0}\left(\mathbf{r}_{1}, \mathbf{X}\right)-L^{-3}\left[\int_{\mathcal{K}} d \mathbf{r} \Psi_{0}(\mathbf{r}, \mathbf{X})\right]\right|^{2}=0
$$


This proves (8), since

$$
\int_{\mathcal{K}^{N-1}} d \mathbf{X} \int_{\mathcal{K}} d \mathbf{r}_{1}\left|\Psi_{0}\left(\mathbf{r}_{1}, \mathbf{X}\right)-L^{-3}\left[\int_{\mathcal{K}} d \mathbf{r} \Psi_{0}(\mathbf{r}, \mathbf{X})\right]\right|^{2}=1-\frac{1}{N L^{3}} \int_{\mathcal{K} \times \mathcal{K}} \gamma\left(\mathbf{r}, \mathbf{r}^{\prime}\right) d \mathbf{r} d \mathbf{r}^{\prime},
$$

and therefore $N^{-1} \gamma_{N} \rightarrow\left|L^{-3 / 2}\right\rangle\left\langle L^{-3 / 2}\right|$ in the limit considered. Moreover, $U\left|L^{-3 / 2}\right\rangle\left\langle L^{-3 / 2}\right| U^{*}$ is given by the kernel $L^{-3} e^{i \varphi\left(z-z^{\prime}\right)}$, so we arrive at (8).

\section{Conclusions}

We have shown that a Bose gas with short range, repulsive interactions is both a $100 \%$ superfluid and also $100 \%$ Bose-Einstein condensed in its ground state in the Gross-Pitaevskii limit where the parameter $N a / L$ is kept fixed as $N \rightarrow \infty$. This is a simultaneous large $N$ and low density limit, because the dimensionless density parameter $\rho a^{3}$ is here proportional to $1 / N^{2}$. If $\rho a^{3}$ is not zero, but small, a depletion of the Bose-Einstein condensate of the order $\left(\rho a^{3}\right)^{1 / 2}$ is expected (see, e.g., [13]). Nevertheless, complete superfluidity in the ground state is not excluded, and is even conjectured by many to be present. It is an interesting open problem to settle this question.

\section{References}

[1] D.R. Tilley and J. Tilley, Superfluidity and Superconductivity, third edition, Adam Hilger, Bristol and New York (1990).

[2] P.C. Hohenberg and P.C. Martin, Microscopic Theory of Superfluid Helium, Ann. Phys. (NY) 34, 291-359 (1965).

[3] The ground state with $\mathbf{v}=0$ remains an eigenstate of the Hamiltonian with arbitrary $\mathbf{v}$ since its total momentum is zero. Its energy is $\frac{1}{2} m N \mathbf{v}^{2}$ above the ground state energy for $\mathbf{v}=0$. Since in a finite box the spectrum of the Hamiltonian for arbitrary $\mathbf{v}$ is discrete and the energy gap above the ground state is bounded away from zero for $\mathbf{v}$ small, the ground state for $\mathbf{v}=0$ is at the same time the ground state of the Hamiltonian with $\mathbf{v}$ if $\frac{1}{2} m N \mathbf{v}^{2}$ is smaller than the gap.

[4] N.V. Prokof'ev and B.V. Svistunov, Two Definitions of Superfluid Density, Phys. Rev. B 61, 11282 (2000).

[5] M. Kobayashi and M. Tsubota, Bose-Einstein Condensation and Superfluidity of a dilute Bose Gas in a random Potential, arXiv:cond-mat/0202364 (2002).

[6] A. Lenard, Momentum distribution in the ground state of the one-dimensional system of impenetrable bosons, J. Math. Phys. 5, 930-943 (1964). 
[7] E.H. Lieb and R. Seiringer, Proof of Bose-Einstein Condensation for Dilute Trapped Gases, Phys. Rev. Lett. 88, \#170409 (2002).

[8] E.H. Lieb and J. Yngvason, Ground State Energy of the Low Density Bose Gas, Phys. Rev. Lett. 80, 2504-2507 (1998).

[9] E.H. Lieb, R. Seiringer, and J. Yngvason, Bosons in a trap: A rigorous derivation of the Gross-Pitaevskii energy functional, Phys. Rev. A 61, 043602-1-13 (2000).

[10] E.H. Lieb and M. Loss, Analysis, second edition, American Mathematical Society $(2001)$.

[11] E.H. Lieb, R. Seiringer, J.P. Solovej, and J. Yngvason, The ground state of the Bose gas, to appear in 'Contemporary Developments in Mathematics 2001', International Press. arXiv:math-ph/0204027 (2002).

[12] E.H. Lieb, R. Seiringer, and J. Yngvason, Poincaré Inequalities in Punctured Domains, arXiv:math.FA/0205088 (2002).

[13] C.J. Pethick and H. Smith, Bose-Einstein Condensation in Dilute Gases, Cambridge University Press (2002). 